\title{
Challenges in generating density perturbations from a fluctuating inflaton coupling
}

\author{
Kari Enqvist \\ Department of Physical Sciences, P. O. Box 64, FIN-00014, University of Helsinki, Finland \\ and Helsinki Institute of Physics, P. O. Box 64, FIN-00014, University of Helsinki, Finland \\ Anupam Mazumdar \\ CHEP, McGill University, Montréal, QC, Canada H3A $2 T 8$ \\ Marieke Postma \\ The Abdus Salam International Centre for Theoretical Physics, I-34100, Trieste, Italy
}

(Received 14 April 2003; published 26 June 2003)

\begin{abstract}
We discuss the possibility of generating adiabatic density perturbations from spatial fluctuations in the inflaton decay rate which are due to quantum fluctuations of light moduli fields coupling to the inflaton. We point out that nonrenormalizable operators, which lift the flatness of the moduli potential, play an important role for the density perturbations. In particular, the nonrenormalizable terms give rise to a considerable damping of the fluctuations and thereby pose an obstruction to the construction of possible models.
\end{abstract}

DOI: 10.1103/PhysRevD.67.121303

PACS number(s): 98.80.Cq

Inflation is the main contender for an explanation of the observed adiabatic density perturbations with a nearly scale invariant spectrum [1]. However, recently alternative mechanisms for generating the density perturbations have also been much discussed. In the curvaton scenario, iscocurvature perturbations of some light "curvaton" field are converted into adiabatic perturbations in the post-inflationary universe [2-4]. Another interesting proposal is that the perturbations could be generated from the fluctuations of the inflaton coupling to the standard model degrees of freedom $[5,6]$. It has been argued that the inflaton coupling strength to ordinary matter, instead of being a constant, could depend on the vacuum expectation value (VEV) of the various fields in the theory. If these fields are light during inflation their quantum fluctuations will lead to spatial fluctuations in the inflaton coupling strength. As a consequence, when the inflaton decays, adiabatic density perturbations will be created because fluctuations in the inflaton coupling translate into fluctuations in the reheating temperature.

A particularly interesting implementation of this scenario is to consider the minimal supersymmetric standard model (MSSM) plus an inflaton field [5]. There are many flat directions in the MSSM [7]. The moduli fields parametrizing these flat directions are light, and their quantum fluctuations during inflation produce fluctuations in the inflaton coupling. The degeneracy of the MSSM scalar potential is lifted by supersymmetry breaking effects and by nonrenormalizable operators. In this note we analyze the effect of these nonrenormalizable operators on the produced density perturbations. We restrict the discussion to MSSM flat directions, but the results can easily be adopted to more general models.

Assuming the inflaton is a gauge singlet, it can decay to normal matter through both normalizable and nonrenormalizable interactions in the superpotential [5]

$$
W \ni \lambda_{h} \phi \bar{h} h+\phi \frac{q}{M} q q+\phi \frac{q_{c}}{M} q_{c} q_{c}+\phi \frac{h}{M} q q_{c},
$$

where $\phi$ is the inflaton, $h$ and $\bar{h}$ are the two Higgs doublets, and $q$ and $q_{c}$ are quark and lepton superfields and their antiparticles. $M$ is some cutoff scale which could be the grand unified theory (GUT) scale or the Planck scale. The effective coupling for inflaton decay into Higgs fields is $\lambda_{h}=\lambda_{0}[1$ $+(S / M)+\ldots]$, where $S$ is the $(\mathrm{VEV})$ of one of the flat direction fields in the theory. The effective coupling for the inflaton decay to quarks is $\lambda_{q}=S / M$ with $S=\langle q\rangle,\left\langle q_{c}\right\rangle$, or $\langle h\rangle$. Effective couplings of this form can result from integrating out heavy particles.

If a single decay channel dominates, the density contrast is $\delta \rho / \rho \sim \delta \lambda / \lambda$. ${ }^{1}$ For $\lambda_{q} \gg \lambda_{h}$ the nonrenormalizable couplings dominate, and the inflaton decays predominantly into quarks and antiquarks. We will refer to this decay as indirect decay. This yields a density contrast

$$
\left.\frac{\delta \rho}{\rho} \sim \frac{\delta S}{S}\right|_{\text {decay }} .
$$

For light fields such that $m_{S}^{2} \ll H^{2}$ the quantum fluctuations are set by the Hubble scale $\delta S \sim H$. The density contrast required to explain the observed temperature anisotropy in the cosmic microwave background (CMB) radiation is $\delta \rho / \rho \sim 10^{-5}$, which can be obtained for $S_{*} \sim 10^{5} H^{*}$ provided there is no later damping of the fluctuations. Here and in the following, the subscript * denotes the corresponding quantity evaluated at the time observable scales leave the horizon, which is some 60 e-folds before the end of inflation. Since $S_{*} \gg H_{*}$ the perturbation spectrum will be Gaussian.

In the opposite limit $\lambda_{h} \gg \lambda_{q}$ the inflaton decays mainly into Higgs fields. We will call this the direct decay channel,

\footnotetext{
${ }^{1}$ It is also possible that one channel is responsible for the fluctuations, but another is the main decay mode. Since the inflaton decay is exponential $\propto \exp (-\Gamma / H)$ it would require some fine-tuning to obtain sizable density perturbations.
} 
as decay is mediated by renormalizable operators. The corresponding density perturbation is

$$
\left.\frac{\delta \rho}{\rho} \sim \frac{\delta S}{M}\right|_{\text {decay }} .
$$

For this decay channel the density perturbations are independent of $S$. The fluctuations will be Gaussian for $S_{*} \gg H_{*}$ and non-Gaussian in the opposite limit. It is important to remember that in Eqs. (2), (3) the right hand side is always evaluated at the time of inflaton decay.

As was pointed out in [5] the flat direction $S$ must have a mass smaller than $H_{*}$ in order to obtain adiabatic, nearly scale invariant, fluctuations. Therefore the Hubble-induced supergravity correction to the moduli mass should not exceed $m_{S} \sim 10^{-1} H_{*}$ in order to have a successful scenario. This can be realized e.g. in no-scale supergravity models [8] with a Heisenberg symmetry imposed on the chiral fields in the Kähler function [9], or in models where inflation is driven by a $D$-term. Apart from the Hubble induced mass terms there are also soft contributions from low energy supersymmetry breaking. However such contributions are small $\sim \mathcal{O}(\mathrm{TeV})$, and do not pose any threat to the scenarios discussed in $[5,6]$. Finally, the flat directions are lifted by nonrenormalizable operators in the superpotential of the form $W$ $=\kappa \mathcal{S}^{n} / n M_{p}^{n-3}[10,11]$. Within the MSSM with $R$-parity conservation most of the flat directions are lifted by $n$ $=4,5,6$ nonrenormalizable operators [11]. The flattest one is lifted by $n=9$. If dominant, these nonrenormalizable contributions can play an important role during and after inflation.

Before discussing the effects of the nonrenormalizable operators, let us start by analyzing the parameter space where they are subdominant and therefore can be neglected. The scalar potential for a flat direction can be written as

$$
V(S)=\frac{1}{2} m_{S}^{2} S^{2}+\frac{\kappa^{2} S^{2(n-1)}}{2^{n-1} M^{2(n-3)}},
$$

where $\kappa \sim \mathcal{O}(1)$, and $m_{S} \sim \mathcal{O}(\mathrm{TeV})$ is the soft mass for the flat direction. We have assumed here that there is no Hubble induced mass correction during inflation. Such a mass term can be included, but it will not change our conclusions in any essential way. Note, however, that for a negative Hubble induced mass ${ }^{2}$, the effective mass can become negative during inflation, $m_{S}^{2}-c H^{2}<0$. The flat direction field then settles in the minimum of the potential, and the nonrenormalizable terms cannot be neglected.

Requiring that the mass term dominates puts an upper bound on the VEV during inflation

$$
S_{*} \lesssim\left(m M^{n-3} / \kappa\right)^{1 /(n-2)} .
$$

For the indirect decay channel $S_{*} \sim 10^{5} H_{*}$, as follows from Eq. (2). This translates into an upper bound on the Hubble constant during inflation: $H_{*} \leq 10^{6}, 10^{8}, 10^{9} \mathrm{GeV}$ for respectively $n=4,5,6$; here we have assumed $M \sim M_{\mathrm{pl}}, \kappa$ $\sim 1$ and $m_{S} \sim \mathrm{TeV}$. However, the bound can be made stronger. Quantum fluctuations during inflation grow until a saturation value $\left\langle S^{2}\right\rangle \approx 3 H^{4} / 8 \pi^{2} m_{S}^{2}$ [12]. If one assumes that the
VEV of $S$ has a typical value $S_{*} \sim \sqrt{\left\langle S_{*}^{2}\right\rangle}$, the bound on the Hubble constant becomes $H_{*} \lesssim 10^{3}, 10^{8}, 10^{10} \mathrm{GeV}$ for $n$ $=4,5,6$ respectively. This result is independent of $m_{S}$, except that $m_{S} \sim 10^{-5} H_{*}$ in order to get density perturbations of the observed size $\delta \rho / \rho \sim 10^{-5}$. Taking $m_{S} \sim \mathrm{TeV}$, this result is inconsistent for $n=4$, i.e., domination of the mass term and density fluctuations of the observed size are incompatible. The results are only marginally consistent for $n$ $=5$.

The density contrast generated through the direct decay channel is independent of $S_{*}$, see Eq. (3). A Gaussian perturbation spectrum requires $H_{*} \leq S_{*}$, with $S_{*}$ bounded by Eq. (5) if the mass term is to dominate.

We will now consider the opposite limit, in which the VEV of $S$ is large and the nonrenormalizable terms in the potential dominate. We should point out that the dynamics of the potential Eq. (4) has already been studied in [2,3] in the context of MSSM curvaton models. A simple analysis shows that during inflation the flat direction field condensate is slow-rolling in the nonrenormalizable potential $V_{N R}$ $\sim \kappa^{2} S^{2(n-1)} / M_{p}^{2(n-3)}$. The amplitude of the field can be estimated as

$$
S_{*} \sim\left(\frac{H M^{n-3}}{\lambda}\right)^{1 /(n-2)}
$$

where $\delta \equiv \delta S / S_{*} \sim H_{*} / S_{*}$.

Now let us turn our attention to the density perturbations. The equations of motion for the homogeneous and the fluctuation parts are given by

$$
\begin{gathered}
\ddot{S}+3 H \dot{S}+V^{\prime}(S)=0 \\
\delta \ddot{S}_{k}+3 H \delta \dot{S}_{k}+\left(\frac{k^{2}}{a^{2}}+V^{\prime \prime}(S)\right) \delta S_{k}=0,
\end{gathered}
$$

where the prime denotes derivative with respect to $S$. Since we are only interested in the long wavelength mode $(k$ $\rightarrow 0$ ), using the slow roll approximation during inflation we get

$$
\begin{gathered}
3 H \dot{S}+V^{\prime}(S)=0, \\
3 H \delta \dot{S}+V^{\prime \prime}(S) \delta S=0,
\end{gathered}
$$

where we have omitted the subscript $k$, understanding that $\delta S$ is for the superhorizon mode. The evolution of the ratio of the fluctuations to the homogeneous mode of $S$ in the nonrenormalizable potential $V_{\mathrm{NR}}$ is

$$
\frac{\delta S}{S} \sim\left(\frac{\delta S}{S}\right)_{\mathrm{i}}\left(\frac{S}{S_{\mathrm{i}}}\right)^{2(n-2)},
$$

where i denotes the initial value. During inflation the zero mode obeys Eq. (9), which can be integrated to yield

$$
\frac{S_{\text {end }}}{S_{*}} \simeq\left(1+\frac{(2 n-4)}{3(2 n-3)} \frac{V^{\prime \prime}\left(S_{*}\right)}{H^{2}} \Delta N\right)^{-1 / 2(n-2)},
$$


with $\Delta N$ the number of e-foldings after observable scales leave the horizon, and $H_{\text {end }}$ the Hubble constant at the end of inflation. In a slow roll regime $V^{\prime \prime}\left(S_{*}\right) \leqslant H_{*}^{2}$. For $\Delta N=60$ we find $S / S_{\mathrm{i}} \sim 0.5$ for $n=4$. We conclude that during inflation the damping of the perturbations is negligible.

After inflation the moduli field $S$ slow-rolls (although marginally) with $V^{\prime \prime} \sim H^{2}(t)$, and we can still use the slow roll approximation Eqs. (9), (10). The perturbations are further damped according to Eq. (11) until $H \sim m_{S}$ and the field starts oscillating in the quadratic potential. There is no further damping during this epoch of oscillations. The adiabatic density perturbations are generated when the inflaton field decays, which happens when $H \sim \Gamma_{\phi} \sim \lambda^{2} m_{\phi}$, with $m_{\phi}$ the mass of the inflaton. ${ }^{2}$ Thus, damping occurs between the end of inflation and, depending on which event happens first, inflaton decay or the onset of moduli oscillations. The total damping factor is

$$
\begin{aligned}
D & \equiv \frac{(\delta S / S)_{\text {decay }}}{(\delta S / S)_{\text {end }}} \sim\left(\frac{\max \left[\Gamma_{\phi}, m_{S}\right]}{H_{\text {end }}}\right)^{2} \\
& \sim \max \left[\lambda_{\text {decay }}^{4},\left(m_{S} / m_{\phi}\right)^{2}\right]
\end{aligned}
$$

\footnotetext{
${ }^{2} \mathrm{We}$ assume that the inflaton decays perturbatively. The results change if preheating occurs, and the inflaton decays through nonperturbative processes.
}

where we have used that at the end of slow roll inflation $H_{\text {end }} \simeq m_{\phi}$. For the indirect decay channel, $\left(\lambda_{q}\right)_{\text {decay }}$ $=S_{\text {decay }} / M$. The correct level of density perturbations is obtained if $D H_{*} / S_{*} \sim 10^{-5}$, as follows from Eq. (2). Assuming $S_{*} \gtrsim H_{*}$, which assures Gaussian fluctuations, implies the limit $S_{\text {decay }} \geq 0.1 M$ or $m_{S} \gtrsim 10^{-2} m_{\phi}$. For the direct decay channel on the other hand $\left(\lambda_{h}\right)_{\text {decay }}=\lambda_{0}$ and density perturbations are of the observed magnitude provided $D H_{*} / M$ $\sim 10^{-5}$, see Eq. (3). For $S_{*} \gtrsim H_{*}$, this requires $\lambda_{0} \gtrsim 0.1$ or $m_{S} \gtrsim 10^{-2} m_{\phi}$.

To conclude, the inclusion of nonrenormalizable operators in the potential of the moduli leads rather generically to a considerable damping of the perturbations. This puts severe constraints on the parameters of the model. In particular, either the inflaton coupling to normal matter should be rather large, $\lambda \gtrsim 0.1$, or the moduli mass should be large, $m_{S}$ $\gtrsim 10^{-2} m_{\phi}$. Another possibility is to consider low scale inflation. In this case the VEV of the moduli is small, and the nonrenormalizable operators in the potential are subdominant. However, for the indirect decay channel we find that this appears to be inconsistent if the potential is lifted by $n$ $=4$ operators, and only marginally consistent for $n=5$. The constraints are milder for the direct decay channel.

A.M. is supported by Cita, K.E. is partially supported by the Academy of Finland grant 51433, and M.P. is supported by the European Union under the RTN contract HPRN-CT2000-00152 Supersymmetry in the Early Universe.
[1] For a review, see A.D. Linde, Particle Physics And Inflationary Cosmology (Harwood Academic, Chur, Switzerland, 1990).

[2] K. Enqvist, S. Kasuya, and A. Mazumdar, Phys. Rev. Lett. 90, 091302 (2003).

[3] K. Enqvist, A. Jokinen, S. Kasuya, and A. Mazumdar, hep-ph/0303165.

[4] K. Enqvist and M.S. Sloth, Nucl. Phys. B626, 395 (2002); D.H. Lyth and D. Wands, Phys. Lett. B 524, 5 (2002); M. Postma, Phys. Rev. D 67, 063518 (2003).

[5] G. Dvali, A. Gruzinov, and M. Zaldarriaga, astro-ph/0303591.

[6] L. Kofman, astro-ph/0303614.

[7] For a review, see K. Enqvist and A. Mazumdar, hep-ph/0209244.
[8] For a review, see A.B. Lahanas and D.V. Nanopoulos, Phys. Rep. 145, 1 (1987).

[9] M.K. Gaillard, H. Murayama, and K.A. Olive, Phys. Lett. B 355, 71 (1995).

[10] M. Grisaru, W. Sigl, and M. Rocek, Nucl. Phys. B159, 429 (1975); N. Seiberg, Phys. Lett. B 318, 469 (1993); M. Dine, L. Randall, and S. Thomas, Nucl. Phys. B458, 291 (1996).

[11] T. Gherghetta, C.F. Kolda, and S.P. Martin, Nucl. Phys. B468, 37 (1996).

[12] T.S. Bunch and P.C. Davies, Proc. R. Soc. London A360, 117 (1978); A.D. Linde, Phys. Lett. 116B, 335 (1982); A.A. Starobinsky, ibid. 117B, 175 (1982); A. Vilenkin and L.H. Ford, Phys. Rev. D 26, 1231 (1982). 